\title{
INDOOR POSITIONING PREDICTION SYSTEM BASED ON WIRELESS NETWORKS AND DEPTH SENSING CAMERAS
}

\author{
Jaime Duque Domingo, Carlos Cerrada \\ jaimeduque@amenofis.com \\ UNED, ETSI Informática, \\ Departamento de Ingeniería de Software y Sistemas Informáticos, \\ C/Juan del Rosal, 16. 28040 Madrid, Spain \\ Enrique Valero \\ Heriot-Watt University, School of Energy, Geoscience, Infrastructure and Society, \\ Edinburgh EH14 4AS, United Kingdom
}

\begin{abstract}
This work presents a new system for predicting the movement of people in indoor user environments, based on an advanced Indoor Positioning System (IPS) developed previously by the authors. The mentioned IPS proposes the combination of WiFi Positioning System (WPS) and depth maps provided by $R G B-D$ cameras to improve the efficiency of existing methods, based uniquely on wireless positioning techniques. In this approach, the prediction of movements is carried out by means of a proactive strategy, delivering the next estimated position of the person. This estimation provides a richer location and context information, which is useful for ubiquitous computing purposes. For example, energy consumption can be optimized if lighting or electronic devices are turned on/off by means of the user trajectory prediction. This paper shows how several techniques, applied for the developed IPS, offer different solutions to the indoor prediction problem, and it discusses about which of them gives better results.
\end{abstract}

Keywords: positioning, WPS, RGB-D sensors, Kinect, WiFi, fingerprint, trajectory, skeletons, depth map, movement prediction, ubiquitous computing

\section{INTRODUCTION}

This work presents a new system for predicting indoor user movements based on an advanced Indoor Positioning System (IPS) [3], and the use of simple prediction techniques [11] [13] [14]. The prediction of the user movements is useful for the development of smart environments, where sustainability principles are required [4] [5]. Since the identity of a user is known in this prediction system, the smart environment would be able to switch on/off lights, control heating or air conditioning systems, or other devices contributing to reduce energy consumption.

In the last years, several works have been developed in the field of mobility prediction. Most of them [7] [15] consider the idea of movements patterns, where user movements are modeled as a repetition of some basic movement patterns. In [7], a pattern matching/recognition-based mobile motion prediction algorithm (MMP) delivers the forthcoming positions of users. However, these algorithms do not deal with abrupt changes in trajectory. Therefore, new solutions focused on these unexpected events should be considered.

In [8], authors explore a novel hierarchical location prediction (HLP) algorithm which is able to deal with random movements. It is composed of a pattern-matching algorithm that extracts any existing regular movement pattern and estimates the global direction between cells (created in a Wireless ATM Network). After this, it uses an extended self-learning Kalman filter that deals with random movements predicting the next cell. Another work [12] uses a Robust Extended Kalman Filter (REKF) to infer an estimation of the next mobile base station where the user's cell phone will be connected. Note that the experiments developed with a car trajectory improve connection reliability and bandwidth efficiency of the underlying system.

Data mining has also been used in movement prediction. Some authors [16] propose a three phases algorithm that firstly obtains mobility patterns from user trajectories by means of data mining, secondly extracts mobility rules from these patterns, and finally calculates predictions using these rules. 
In ubiquitous computing [10], active strategies predict user movement through trajectories and locations in order to provide richer location and context information. This work presents two models for trajectory prediction, namely probabilitybased model and learning-based model. The probability based model stores long-term historical trajectories for probability calculation and trajectory prediction.

Finally, in [2], authors propose a mixed Markovchain model (MMM) that considers the pedestrian's personality as an unobservable parameter. They also consider the pedestrian's previous status. Their experiments have been performed in a major shopping mall where they have demonstrated the highest prediction accuracy of their method.

This work makes a review about different prediction methods and how they are used with the IPS previously developed by the authors. It also evaluates the results obtained.

This work is structured as follows: First of all, the IPS based on WiFi networks and RGB-D cameras section explains how the IPS developed in [3] works. Secondly, the Movement prediction section explores several techniques for movement prediction by means of the previously described IPS. Experimental results obtained by applying some of these techniques are also included and discussed in this section. Finally, the Conclusions section remarks the main features of the presented system.

\section{IPS BASED ON WIFI NETWORKS AND RGB-D CAMERAS}

Indoor positioning can be easily improved with the combination of WPS and RGB-D cameras. The system presented in [3] just requires smart-phones, routers, and the installation of RGB-D cameras, while other positioning systems force users to wear non-common devices or cards on their bodies. Figure 1 shows how this system works. Each user carries their portable device, i.e. smart phone, which scans the RSSI signals received from all reachable routers. These data are synchronously sent to a server which obtains a depth map using the RGB$\mathrm{D}$ camera. This server extracts the skeletons of the users and relates each skeleton to each portable device. This process is performed by minimizing the Euclidean distance between each skeleton and the position of the user according to WPS fingerprinting.

WPS fingerprinting is a common technique used

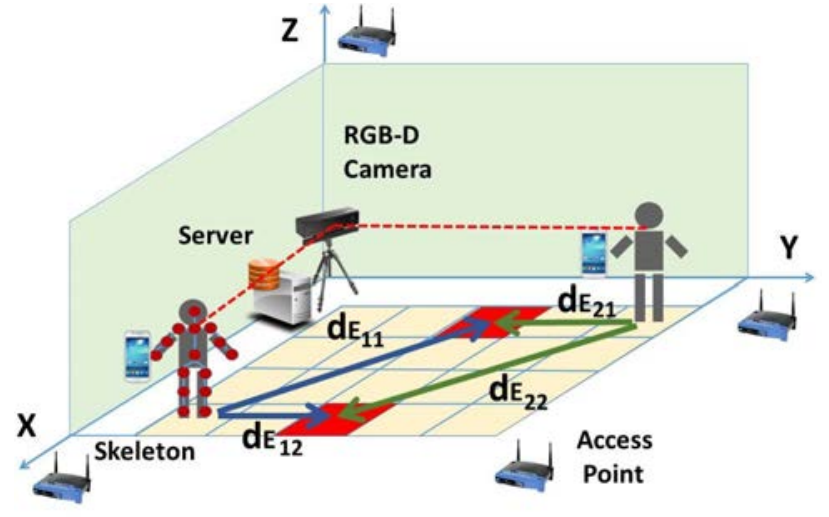

Figure 1: System scheme for indoor positioning

for indoor positioning based on the use of wireless devices, such as mobile phones, to measure the signal level they receive from various access points (A.P.), i.e. WiFi routers. Most current WPSs use the technique named fingerprinting [9]. With this technique, a map is created by recording the different RSSI values received at each point. Tables 1 and 2 show how a fingerprint map is built, relating a group of coordinates $\mathrm{X}, \mathrm{Y}$ and $\mathrm{Z}$ to RSSI values received by the portable device from several APs. The obtained values deliver the position of a user in real-time, comparing the values received from its wireless device to the values stored on the map.

Table 1: Table of positions.

\begin{tabular}{|c|l|l|l|}
\hline id. & $\mathbf{X}$ & $\mathbf{Y}$ & $\mathbf{Z}$ \\
\hline 1 & 0,2986 & 0,0358 & 1,2372 \\
2 & 0,2295 & 0,0252 & 1,7389 \\
3 & 1,0071 & 0,0452 & 0,8489 \\
\hline
\end{tabular}

Table 2: Example of fingerprint map.

\begin{tabular}{|c|l|l|l|}
\hline id. & BSSID & Router & RSSI \\
\hline 1 & dc:53:7c:25:2c:36 & Router 1 & -53 \\
1 & 84:9c:a6:fe:0e:34 & Router 2 & -46 \\
1 & 4c:54:99:df:9e:ec & Router 3 & -93 \\
2 & dc:53:7c:25:2c:36 & Router 1 & -74 \\
2 & 84:9c:a6:fe:0e:34 & Router 2 & -40 \\
2 & 4c:54:99:df:9e:ec & Router 3 & -73 \\
3 & dc:53:7c:25:2c:36 & Router 1 & -52 \\
3 & 84:9c:a6:fe:0e:34 & Router 2 & -51 \\
3 & 4c:54:99:df:9e:ec & Router 3 & -89 \\
\hline
\end{tabular}

An advanced indoor positioning method based on the combination of WPS and RGB-D consists in considering the user trajectory. Two different datasets are considered to estimate the trajectory of users. The first set includes the last points where the user has been situated according 
to WPS and the second set contains the last areas visited by the person according to RGB-D data.

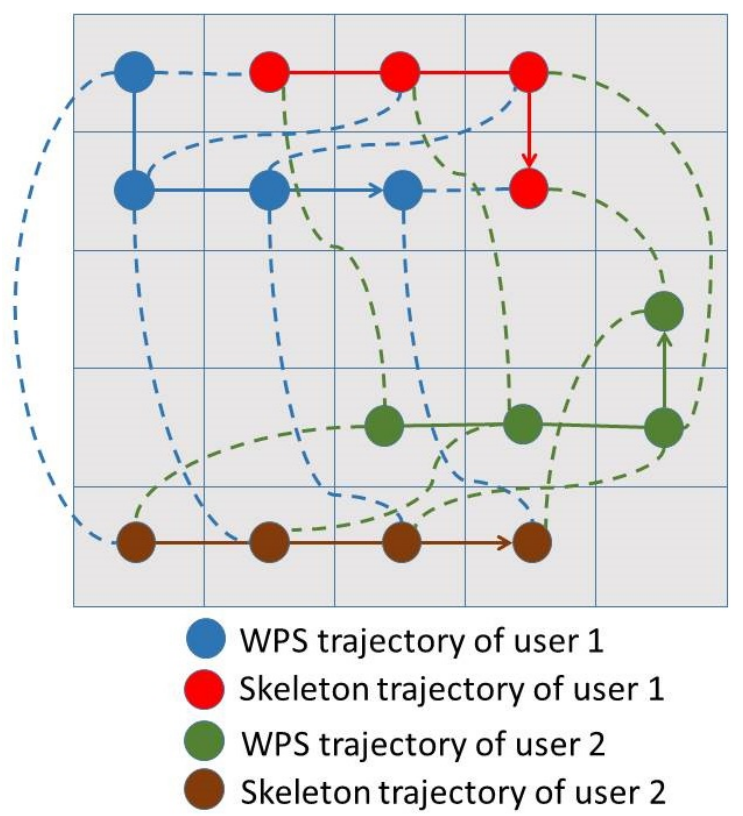

Figure 2: User trajectories

Figure 2 illustrates the position of two users in the last four instants of time, named time stamps. Each user is related to a portable device by means of the minimum sum of the Euclidean distances between each skeleton position and the WPS position for the different trajectories. The dashed lines represent the calculated Euclidean distances. As mentioned in [6], the Synchronized Euclidean distance measures the distance between two points at identical time stamps. If two trajectories with different points are obtained at the same time (for each pair), the total error is measured as the sum of the distances between all points (points in WPS trajectory and points in skeleton trajectory) at synchronized time stamps.

This advanced IPS system, considering the user trajectories, delivers results with success rates over $70 \%$ when locating three users in a small room of $4.5 \times 4.5$ meters. When there are two users, results are successful in above $89 \%$ of the cases.

Once users have been located, it is possible to obtain a prediction of their trajectories. RGB-D provides a more accurate trajectory than WPS, since the positioning error is lower. The combination between RGB-D and WPS relates each user to a portable device so the user identity is known. Therefore, this method allows discerning the RGB-D trajectory to a known identity user.

\section{MOVEMENT PREDICTION}

Since skeleton trajectory is known with RGB-D, it is possible to estimate a prediction of the user movements. This prediction is useful for detecting where the user goes and, therefore, being able to open/close a door, switch a light on/off or turn the heater on/off according to user preferences. Although user movements may sometimes seem random, several techniques help calculate accurate predictions. These methods are based on the user trajectory, taking into account the last points where the user has been. These methods include regression [11] or Kalman filter [14]. Other techniques use the knowledge about user movements, like neural networks [13]. A Bayesian probability model with a neural network is proposed in [1] while movement patterns are considered in [7], [8] and [15].

Regression is a technique that allows obtaining a function for approaching the last points of a trajectory. Following that function, it is possible to predict next positions. The user trajectory can be defined as a set of points $P(x, y)$, where each axis can be expressed as a time function: $X(t)$ and $Y(t)$. The regression approaches each function, applied for each axis $X(t)$ and $Y(t)$, combining the predictive values as a new point $P\left(X\left(t^{\prime}\right), Y\left(t^{\prime}\right)\right)$, where $t^{\prime}$ is the next time stamp.

Kalman filter and Extended Kalman filter [14] are well known techniques that allow predicting the next user position. This filter considers noise and other inaccuracies and helps rectify the signals, giving the possibility to obtain a prediction. Kalman filter uses Bayesian inference and estimates a joint probability distribution over the variables for each time stamp. The initial state vector includes the coordinates of the user and the displacement between consecutive time stamps.

Neural networks use the knowledge of the past user movements. Since users usually follow a similar path, a neural network helps predict the next movement. In [13], a neural network is used for trajectory prediction for moving objects. During the learning stage, the entrance layer of the network represents the displacements between the last user positions, for each axis $\mathrm{X}$ and $\mathrm{Y}$. The exit layer of the network represents the displacement between the last two positions of the user. If five time stamps are considered for learning, the entrance cells consists in $\Delta p_{x}(t-4, t-3), \quad \Delta p_{y}(t-4, t-3)$, $\Delta p_{x}(t-3, t-2), \quad \Delta p_{y}(t-3, t-2) \quad$, $\Delta p_{x}(t-2, t-1)$ and $\Delta p_{y}(t-2, t-1)$, while the exit cells are $\Delta p_{x}(t-1, t)$ and $\Delta p_{y}(t-1, t)$. During the operation stage, the entrance of the 
network consists in the displacements between the last four user positions (four time stamps), while the exit returns the predicted displacement of the user. That displacement is added to the last user position for obtaining the next prediction of user position. Figure 3 represents the neural network used in the experiments.

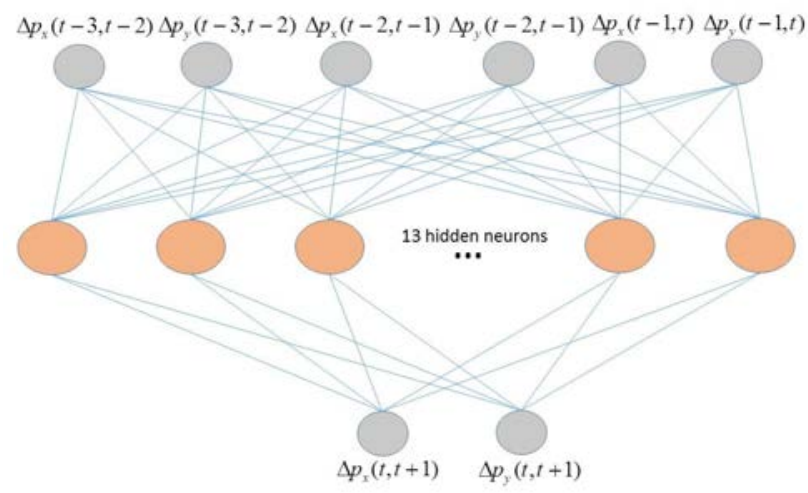

Figure 3: Neural network for trajectory prediction used in the experiment

\subsection{Experiments and results}

Several experiments have been performed using linear and polynomial regression, Kalman filter, and the use of neural networks. The experiments consist in determining the next user position considering the last four positions obtained in four timestamps where the user has been situated. The position is established by means of the skeleton coordinates obtained by the RGB-D camera. Speed and acceleration of the user has not been taken into account since the position implicitly is related to them.

To simplify the positioning and prediction process, the floor of the working area is divided according to [3], where after obtaining the fingerprint map, 25 cells are created ( 5 x 5 square cells of 0.74 meters side). The division in cells allows to obtain the user's position easily and is used for the prediction process. In this way, the system predicts in which cell the user will be located during the next time stamp.

With regard to the prediction problem, the first techniques considered are those related to regression. Simple linear regression adjusts data to a function with the form of Equation 1 while polynomial regression with degree 2 adjusts data to Equation 2 and polynomial regression with degree 3 adjusts to Equation 3. Each axis has been considered independently for regression process as explained before.

$$
y=a+b x
$$

$$
y=a+b x+c x^{2}
$$

$$
y=a+b x+c x^{2}+d x^{3}
$$

For the Kalman filter, the initial state vector includes the coordinates of the user and the displacement between consecutive time stamps. Instead of using the speed, the displacement between consecutive user positions has been considered.

A neural network based on a multilayer perceptron has been built (see Figure 3) where there is one input layer with 6 neurons, one hidden layer with 13 neurons and one exit layer with 2 neurons. A bipolar sigmoid activation function has been employed and the learning rule is based on backpropagation with momentum. 200 user coordinates have been recorded to train the network, consisting in several movement patterns that users make. With them, other predicted movement patterns have been added to the data base for the learning process. As explained before, the entrance layer represents the displacements in the axis between two consecutive time stamps. The exit layer represents the displacements between the last two positions in the learning or between the last one and the prediction during usual operation of the network. The results of the experiments have been shown using the cell division created during WPS with RGB-D positioning since this work is a continuation of the positioning system based on those technologies [3].

Figure 4 shows five situations where several skeleton trajectories are represented. The arrows with discontinued lines point the obtained predictions with the different methods. The cells in lighter green represent the real position of the user at the prediction time. For example, the real trajectory of the user 4 matches with the predicted position of the different methods, cell $(5,1)$. In some cases, the methods obtain a prediction outside the working area. Polynomial regression with degree 3 produces outliers while the other methods produce more accurate predictions. In these five situations, neural networks offer the best results.

If there are static obstacles in the work space that impede the user movement, like a table or a locker, neural networks offer better predictions because they only learn possible real movements. During 
the learning, users avoid the obstacles. Kalman filter and regression do not take into account the obstacles and are not able to deal with them. However, Kalman filter and regression do not need the learning process and, therefore, they are easier to implement.

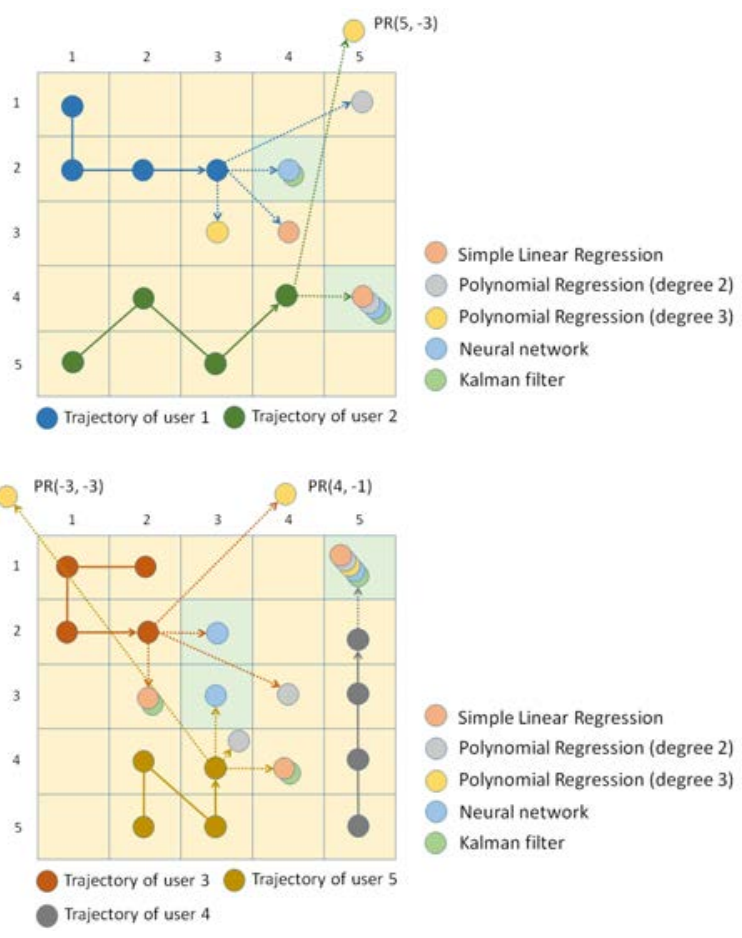

Figure 4: Results of skeleton trajectory prediction

When there are dynamic obstacles in the work space, like a chair or a box, none of these methods will avoid the obstacles. RGB-D camera allows obtaining some possible obstacles, being necessary to combine this information with the prediction system in order to obtain a more accurate position and avoid the static and dynamic obstacles.

The results of the experiments are shown in Table 3. 25 different tests have been carried out.

Table 3: Successes in indoor movement prediction.

\begin{tabular}{|c|l|}
\hline Technique & Successes \\
\hline Simple linear regression & $76 \%$ \\
Polynomial regression (degree 2) & $80 \%$ \\
Polynomial regression (degree 3) & $36 \%$ \\
Kalman filter & $84 \%$ \\
Neural network & $92 \%$ \\
\hline
\end{tabular}

\section{CONCLUSIONS}

The results presented in this work show how several techniques are applied for the prediction problem. Using an advanced indoor positioning system, these techniques help predict user move- ments. Between these techniques, neural networks offer more accurate results. These predictions deliver a richer location which increases context awareness. In ubiquitous computing, proactive strategies like user movement predictions, offer a solution to high energy consume and improve user experience. This work uses an IPS that just requires smart phones, widely extended, and that is based on the combination of WiFi positioning system and RGB-D cameras.

Next developments will be oriented towards the use of this information for ubiquitous computing purposes. Little by little, ubiquitous computing is being introduced in life. The advances in smart phones, networks, sensors and cameras contribute to create a more awareness world where user movements and their predictions play a key role.

\section{Acknowledgments}

This work has been developed with the help of the research project DPI2013-44776-R of MICINN. It also belongs to the activities carried out within the framework of the research network CAM RoboCity2030 S2013/MIT-2748 of Comunidad de Madrid.

\section{References}

[1] Akoush, Sherif and Sameh, Ahmed, (2007) Mobile user movement prediction using bayesian learning for neural networks, Proceedings of the 2007 international conference on Wireless communications and mobile computing, ACM, pg. 191-196.

[2] Asahara, Akinori and Maruyama, Kishiko and Sato, Akiko and Seto, Kouichi, (2011) Pedestrian-movement prediction based on mixed Markov-chain model, Proceedings of the 19th ACM SIGSPATIAL international conference on advances in geographic information systems, ACM, pg. 25-33.

[3] Duque Domingo, Jaime and Cerrada, Carlos and Valero, Enrique and Cerrada, JA, (2016) Indoor Positioning System Using Depth Maps and Wireless Networks, Journal of Sensors, Hindawi Publishing Corporation.

[4] GhaffarianHoseini, AmirHosein and Dahlan, Nur Dalilah and Berardi, Umberto and GhaffarianHoseini, Ali and Makaremi, Nastaran, (2013) The essence of future smart houses: From embedding ICT to adapting to sustainability principles, Renewable and Sustainable Energy Reviews, Elsevier pg. 593-607, vol. 24.

[5] Kok, Koen and Karnouskos, Stamatis and Nestle, David and Dimeas, Aris and Weidlich, 
Anke and Warmer, Cor and Strauss, Philipp and Buchholz, Britta and Drenkard, Stefan and Hatziargyriou, Nikos and others, (2009) Smart houses for a smart grid, Electricity Distribution-Part 1, 2009. CIRED 2009. 20th International Conference and Exhibition on, IET, pg 1-4.

[6] Lawson, Catherine $\mathrm{T}$ and Ravi, SS and Hwang, Jeong-Hyon, (2011) Compression and Mining of GPS Trace Data: New Techniques and Applications, Technical Report. Region II University Transportation Research Center.

[7] Liu, George Y and Maguire, GQ, (1995) A predictive mobility management algorithm for wireless mobile computing and communications, Universal Personal Communications. 1995. Record., 1995 Fourth IEEE International Conference on, IEEE, pg. 268-272.

[8] Liu, Tong and Bahl, Paramvir and Chlamtac, Imrich, (1998) Mobility modeling, location tracking, and trajectory prediction in wireless ATM networks, Selected Areas in Communications, IEEE Journal on, pg. 922-936, vol. 16.

[9] Liu, Wei and Chen, Yongle and Xiong, Yongping and Sun, Limin and Zhu, Hongsong, (2014) Optimization of Sampling Cell Size for Fingerprint Positioning, International Journal of Distributed Sensor Networks, Hindawi Publishing Corporation.

[10] Liu, Xiong and Karimi, Hassan A, (2006) Location awareness through trajectory prediction, Computers, Environment and Urban Systems, Elsevier, pg. 741-756, vol. 30.

[11] Neter, John and Kutner, Michael H and Nachtsheim, Christopher J and Wasserman, William, (1996) Applied linear statistical models, Irwin Chicago, vol. 4.

[12] Pathirana, Pubudu N and Savkin, Andrey V and Jha, Sanjay, (2004) Location estimation and trajectory prediction for cellular networks with mobile base stations, Vehicular Technology, IEEE Transactions on, pg. 19031913, vol. 53 .

[13] Payeur, Pierre and Le-Huy, Hoang and Gosselin, Clement M, (1995) Trajectory prediction for moving objects using artificial neural networks, Industrial Electronics, IEEE Transactions on, pg. 147-158, vol. 42.

[14] Rosales, Romer and Sclaroff, Stan, (1998) Improved tracking of multiple humans with trajectory prediction and occlusion modeling, IEEE CVPR workshop on the Interpretation of Visual Motion.

[15] Tabbane, Sami, (1995) An alternative strategy for location tracking, Selected Areas in Communications, IEEE Journal on, IEEE, pg. 880-892, vol. 13 .

[16] Yavaş, Gökhan and Katsaros, Dimitrios and Ulusoy, Özgür and Manolopoulos, Yannis, (2005) A data mining approach for location prediction in mobile environments, Data \& Knowledge Engineering, Elsevier, pg. 121146, vol. 54 . 